\title{
Modelo de juego serio colaborativo basado en agentes inteligentes para apoyar procesos virtuales de aprendizaje
}

\author{
Santiago Álvarez, Oscar M. Salazar y Demetrio A. Ovalle \\ Universidad Nacional de Colombia, Departamento de Ciencias de la Computación y de la Decisión, \\ Facultad de Minas, Medellín-Colombia (correo-e: salvarezl@unal.edu.co, omsalazaro@unal.edu.co, \\ dovalle@unal.edu.co)
}

Recibido Dic. 26, 2019; Aceptado Feb. 28, 2020; Versión final Abr. 23, 2020, Publicado Oct. 2020

\begin{abstract}
Resumen
El objetivo de este artículo es proponer y validar un modelo de juego serio colaborativo que permita mejorar la interacción entre equipos de trabajo e incentivar la participación de los estudiantes. El juego serio propone un enfoque lúdico para evaluación de conocimientos de los estudiantes trabajando en equipos, el cual permite la identificación de fallas cognitivas. Se construyó un prototipo de juego serio denominado COLEGA (Collaborative Learning Game) que se validó en un ambiente controlado al interior del curso de inteligencia artificial de la Facultad de Minas de la Universidad Nacional de Colombia - Sede Medellín. Los resultados muestran la aceptación por parte de los estudiantes al utilizar entornos computacionales que integran enfoques lúdicos y colaborativos para mejorar el aprendizaje. Se concluye que el modelo de juego serio basado en agentes inteligentes y aprendizaje colaborativo brinda mecanismos que motivan la colaboración y participación en la actividad.
\end{abstract}

Palabras clave: juego serio colaborativo; aprendizaje colaborativo; agentes inteligentes; computación sensible al contexto

\section{An intelligent-agent-based collaborative serious game model to support virtual learning processes}

\begin{abstract}
The aim of this paper is to propose and validate a model of a collaborative serious game that improves teamwork interactions and that encourages student participation. The serious game proposes a gamification approach to assess knowledge of students working in teams and to identify cognitive failures. In order to validate the proposed model, a serious game prototype called COLEGA (Collaborative Learning Game) was built and tested with a case study in a controlled environment: the artificial intelligence course at the Faculty of Mines of the National University of Colombia (Medellin). The results show student acceptance of computational environments that integrate gamification approaches along with collaborative techniques to improve their learning. It is concluded that the serious game model, based on intelligent agents and collaborative learning, provides mechanisms that motivate collaboration and activity participation.
\end{abstract}

Keywords: collaborative serious game; collaborative learning; intelligent agents; context-aware computing 


\section{INTRODUCCIÓN}

Es evidente el relacionamiento de los miles de juegos existentes en el mercado con una tendencia orientada hacia el entretenimiento, sin embargo, es importante destacar que los juegos tradicionales no se centran en el estímulo de aprendizaje, solo en el deleite y la diversión (Aguilera et al., 2014). A partir de esto, los juegos serios aparecen como una alternativa viable a este tipo de problemas porque son sistemas que buscan, en primer lugar, motivar y entretener a los estudiantes mientras brindan adicionalmente un carácter educativo para lograr objetivos de aprendizaje, resolver problemas, aprender un tema específico o desarrollar habilidades. Lo anterior, con el objetivo de siempre intentar el mejoramiento del aprendizaje (Acevedo et al., 2010). Los juegos serios se están construyendo en diferentes niveles de educación tanto en las escuelas primarias y secundarias como en las universidades de todo el mundo (Terzidou et al., 2016). Adicionalmente, según (Hernández et al., 2014) se ha comprobado que las nuevas generaciones tienen más desarrollado el canal visual, y poseen una alta estima hacia lo lúdico. Lo anterior gracias a la exposición desde temprana edad a las nuevas tecnologías de la información y comunicación. Sin embargo, según Hainey et. al (2013) los juegos serios no necesariamente fomentan un compromiso y un aprendizaje más profundos; por lo tanto, se requiere trabajar específicamente en el diseño instruccional basado en el juego.

El aprendizaje colaborativo o CL (por sus siglas en inglés para Collaborative Learning) se define como una técnica de aprendizaje en la cual el principal objetivo es potenciar el aprendizaje individual (Yannibelli \& Amandi, 2011) a través de la conformación de un conjunto de personas trabajando de forma organizada y en busca de un mismo objetivo. Adicionalmente, dicha modalidad de aprendizaje puede ejecutarse a través del uso de comunicaciones virtuales, es así como aparece el aprendizaje colaborativo apoyado por computador - CSCL (por sus siglas en inglés para Computer Supported Collaborative Learning), que se define como un campo de investigación dirigido a la "construcción del conocimiento o la resolución de problemas a través del acoplamiento mutuo de dos o más estudiantes en un esfuerzo coordinado a través de herramientas computacionales, internet y comunicaciones electrónicas para sus interacciones" (Diaz, Brown, \& Salmons, 2010). Por otra parte, los agentes inteligentes corresponden a entidades computacionales autónomas y con capacidad para ejecutar tareas y lograr sus metas con un mínimo de supervisión humana o incluso ninguna.

Para Caicedo y Weiss (2011) son características que deberían poseer los agentes para trabajar efectivamente (Carrera et al., 2014): a) reactividad: responden inmediatamente a los cambios percibidos en su entorno; b) distribución de tareas: cada agente tiene funcionalidades bien definidas e identifica los problemas a resolver; c) proactividad: los agentes toman iniciativa para la solución de problemas; d) cooperación y coordinación: realizan tareas por medio de intercambio de mensajes con otros agentes a través de un lenguaje común; e) autonomía: los agentes no requieren de la intervención directa de los seres humanos para operar; f) deliberación: cada agente tiene la capacidad de realizar procesos de razonamiento interno los cuales le permitan tomar decisiones; g) movilidad: pueden moverse de un nodo a otro a través de la red; h) adaptación: a partir de los cambios en el entorno cambian su comportamiento y mejoran de esta forma su desempeño, y finalmente i) paralelismo: el sistema puede mejorar su desempeño a través de la ejecución de tareas realizadas en paralelo por los agentes.

De esta forma, los sistemas multi-agente (SMA) se conforman a partir de un conjunto de agentes inteligentes que trabajan conjuntamente e interactúan coordinadamente en un entorno computacional para resolver problemas específicos y de alta complejidad (Elimelech et al., 2017). Según Ovalle et al. (2014) este tipo de sistemas ofrecen una gran facilidad de adquisición y procesamiento de información que puede encontrarse altamente distribuida. De esta manera, este paradigma ofrece nuevas maneras para analizar, diseñar e implementar sistemas de software complejos (Lhafiane et al., 2015).

Adicionalmente, el concepto de servicios de Concienciación, se usa en entornos de aprendizaje, sobretodo, con el fin de proporcionar a un aprendiz la consciencia del contexto durante el desarrollo de una actividad de aprendizaje. Es allí donde surgen los servicios de Concienciación (Alzaza y Yaakub, 2011), que básicamente permiten conectar el contexto con un sistema informático a partir de la recopilación de datos, brindando características de sensibilidad y consciencia del contexto al sistema. De esta manera, dicho aprendiz, quien bien puede ser considerado como un estudiante, se sensibiliza no solo del desempeño de su trabajo individual, sino que al mismo tiempo crea consciencia del apoyo que debe brindar al desempeño de su equipo de trabajo (en caso de estar inmerso en un entorno de aprendizaje colaborativo). Ahora bien, en los entornos colaborativos, es frecuente que los estudiantes no puedan ver, escuchar, o ni siquiera llegar a percibir cada una de las acciones de su compañero de trabajo. En este tipo de entornos, estas habilidades de conciencia de trabajo en equipo son bastante limitadas. En este sentido, la consciencia del contexto y la sensibilidad sobre el mismo, se ha convertido en uno de los temas principales en el diseño de sistemas informáticos de aprendizaje con el fin de reducir la necesidad de los esfuerzos meta-cognitivos para colaborar en entornos informáticos distribuidos. 


\section{OTROS ANTECEDENTES}

A continuación, se presentan algunos trabajos relacionados que permiten conocer los modelos, herramientas y enfoques actuales en el campo de investigación, permitiendo así contrastar las bondades del modelo propuesto en esta investigación. Samadi et al. (2018) proponen un sistema de preguntas y respuestas QAS (por sus siglas en inglés para Question Answering System) el cual posee un enfoque con agentes sintácticos y semánticos para responder preguntas en plataformas de aprendizaje en línea (e-learning). Dicha propuesta se basa en mejorar los enfoques existentes a partir de la generación de una arquitectura compuesta por tres capas: (1) capa de usuario, (2) capa de búsqueda de respuestas y (3) capa de base de datos. Primero, la capa de usuario recibe la pregunta (vocal o de texto) y la envía a la capa de búsqueda de respuestas, donde diferentes agentes analizan la pregunta y buscan la mejor respuesta de todos los recursos disponibles (banco de preguntas y respuestas, cursos BD, Internet) y la comunican con el profesor u otros alumnos si es necesario. En detalle, el QAS propuesto i) recibe la pregunta del alumno y la lleva a la fase de análisis, donde se realiza un análisis sintáctico y semántico sobre la pregunta para definir su naturaleza, tipo y otras propiedades, utilizando el procesamiento del lenguaje natural, se intenta analizar la pregunta y presentarla en forma de ontologías pedagógicas, posteriormente, ii) el sistema verifica si la pregunta ya ha sido respondida (de la base de datos de Preguntas / Respuestas), si hay una respuesta, la envían al alumno, de lo contrario iii) se inicia un proceso de búsqueda de respuestas, donde diferentes agentes trabajan juntos en varias fuentes y bases de datos para encontrar la mejor respuesta. Si la respuesta no se puede encontrar automáticamente, otros tutores y alumnos contribuyen a responder la pregunta. Es importante destacar que con la inclusión y la cooperación entre los agentes los autores buscan alcanzar las mejores soluciones en cada paso del procesamiento a la vez que dotan de adaptabilidad, interactividad, distribución, colaboración, personalización, seguridad e inteligencia al modelo, sin embargo, el sistema únicamente se enfoca en buscar respuestas de preguntas, lo cual no genera en gran medida interacción entre los estudiantes.

Bennane (2013) propone el sistema multi-agente SMAASA el cual integra lógica difusa para la automatización del apoyo de los alumnos en una plataforma de aprendizaje colaborativo en línea (Collaborative e-learning Platform). La idea principal de los autores es aprovechar los datos de interacción entre los alumnos durante el proceso de aprendizaje para mejorar el nivel de colaboración. Posteriormente, dichos datos son analizados y almacenados como indicadores en el perfil del alumno, y basado en estos indicadores, el sistema evalúa el estado de la colaboración de los alumnos y envía recomendaciones automáticas para mejorar la colaboración. Teniendo en cuenta la naturaleza imprecisa de la información que es manipulada (datos de actividades del alumno), la automatización del proceso de evaluación del comportamiento colaborativo del alumno, llevó a los autores a dotar a los agentes del sistema de características difusas, ya que la lógica difusa es una solución adecuada para manejar imprecisiones e incertidumbres. El sistema calcula los indicadores: número de mensajes, número de discusiones, número de conexiones realizadas y, por lo tanto, evalúa el grado de colaboración, el grado de presencia y el nivel de participación de cada alumno en su grupo, asignando una puntuación entre 0 y 20 . El experimento realizado para probar el sistema mostró que los alumnos reaccionaron positivamente a las recomendaciones enviadas, lo que influye en la evolución efectiva en el nivel de participación de los alumnos. A partir de lo anterior, se puede destacar que una de las fortalezas de esta propuesta es que se enfoca en mejorar el comportamiento colaborativo de los estudiantes al interior de una actividad de aprendizaje colaborativo en línea incluyendo lógica difusa para manejar la incertidumbre. Por otro lado, se evidencia que el sistema omite causas importantes que pueden generar desmotivación y poca interacción en el equipo por parte de los estudiantes, dejando también de lado el contexto sobre el cual se desempeña la actividad.

Briede et al. (2015) utilizan una Pizarra Digital Interactiva (PDI) para apoyar los procesos de enseñanzaaprendizaje colaborativos de forma que sea esta una plataforma de proyección pública y pantalla interactiva para estudiantes y profesores. Adicionalmente, se garantiza la construcción de un registro de aprendizaje a través del almacenamiento digital, el cual aporta a la sociabilización entre grupos de estudiantes y favorece de esta forma la retroalimentación de los conceptos, croquis y proceso creativo para un curso de diseño industrial. Por su parte (Hernández-Horta el al., 2018) proponen el diseño de un juego basado en los principios de Gamificación, cuyo fin es crear y modificar actitudes y aptitudes dentro de los individuos involucrados en el juego, para Instituciones de Educación Superior (IES) en la Ciudad de México. El objetivo del juego es desarrollar competencias en los estudiantes y complementar los procesos de enseñanza-aprendizaje tradicionales. Los autores utilizan una metodología genérica la cual puede adaptarse a cualquier rama de la ciencia y a las demandas de cada profesor en específico, sin dejar de lado en desarrollo de competencias básicas. De esta forma, la metodología incluye las siguientes etapas: 1) Definir los objetivos buscados en el aula, 2) Determinar los comportamientos deseados acorde a la literatura de aprendizaje basado en juegos, 3) Describir el tipo de jugadores (estudiantes) hacia los cuales va dirigido el juego, 4) Elegir las actividades de aprendizaje individual o en grupo a realizar, y 5) Desarrollar herramientas TICs a utilizar y especificar sus requerimientos. Los autores al finalizar los dos primeros pasos de la metodología obtuvieron varios diagramas, los cuales fungen como base teórica y esquemática para la elaboración de la propuesta del juego. 
González-González et al. (2019) construyen un sistema inteligente de rehabilitación basado en el videojuego de ejercicio TANGO:H (proveniente de Tangible Goals: Health) la cual puede ser usada para apoyar el proceso de rehabilitación de niños hospitalizados. La propuesta consta de dos elementos: una plataforma inteligente con el videojuego y una herramienta de diseño de ejercicios. La plataforma inteligente incluye un sistema de recomendación que analiza las interacciones del usuario, junto con el historial del usuario, para seleccionar nuevos ejercicios gamificados para el usuario. Una de las principales contribuciones de este trabajo se centra en definir un sistema de recomendación basado en diferentes niveles de dificultad y habilidades del usuario para ofrecer la posibilidad de proporcionar al usuario un modo de juego personalizado basado en su propio historial y preferencias. Según los autores uno de los principales problemas de la rehabilitación es que las sesiones de terapia pueden ser aburridas ya que normalmente consisten en repetir los mismos ejercicios una y otra vez. Por lo anterior, los juegos pueden ayudar a mejorar la motivación y hacer que los pacientes trabajen más (Proffitt et al., 2015; Rego et al., 2014).

Los módulos principales de TANGO: $\mathrm{H}$ son: (a) Juego TANGO: $\mathrm{H}$, donde los jugadores pueden interactuar con el sistema; (b) Diseñador TANGO: $\mathrm{H}$, donde los profesionales pueden diseñar diferentes tipos de ejercicios, diseñar la gamificación de ejercicios, crear usuarios definiendo sus características y habilidades, y asignar ejercicios a usuarios o grupos particulares; (c) el modelo de usuario con el perfil de usuario, habilidades, nivel, interacciones y gamificación; y (d) el sistema de recomendación, con las reglas de personalización y recomendación que son presentadas a los jugadores. Las principales contribuciones de este trabajo son la creación de módulos inteligentes para TANGO: $\mathrm{H}$ y la evaluación de la efectividad, eficacia, capacidad de aprendizaje y satisfacción de los ejercicios basados en gestos. Las nuevas características desarrolladas incluyen el nivel del sistema basado en habilidades y la recomendación personalizada. Uno de los puntos a destacar de esta propuesta es que propone un juego serio con ejercicios de realidad virtual que son personalizados para cada jugador, sin embargo, sería importante incluir agentes inteligentes para agregar distribución, inteligencia y proactividad al modelo.

Mizukoshi et al. (2016) proponen una herramienta de aprendizaje colaborativo soportado por computador para cursos escolares de niveles inferiores. El estudio se centra en validar la usabilidad de la herramienta y en aclarar la contribución que esta tiene en el proceso de enseñanza a partir del despliegue de las funcionalidades. El objetivo de la aplicación es que los estudiantes por medio de dispositivos móviles —en este caso tablets- puedan interactuar entre ellos y llevar a cabo una serie de actividades que se despliegan en el tablero. Algunas de las actividades son: realizar pantallazos, seleccionar asignaturas favoritas y sustentarlo, tomar fotos de elementos pertenecientes a la clase, realizar cuestionarios, etc. La herramienta se apoya en un modelo colaborativo, que permite la difusión de resultados por parte de los estudiantes, lo que fomenta la discusión argumentativa. La herramienta corresponde a un sistema móvil que apoya el aprendizaje colaborativo y puede crear una red inalámbrica autónoma sin usar un punto de acceso de red inalámbrica externo, permitiendo conectarse a Internet a través de tablets. Además, este permite compartir información entre estudiantes y profesor de manera muy sencilla a través de la visualización de las diferentes pantallas de las tablets desde una sola. Sin embargo, la investigación no considera aspectos de detección de fallas, ni presenta una estructura de conocimiento explícita que permita describir mejor el modelo utilizado. Por otra parte el sistema no involucra elementos de recomendación para mejorar el proceso de aprendizaje ni se enfoca en motivar a los alumnos a utilizar la herramienta propuesta, lo que puede generar aburrimiento en los estudiantes.

Ee et al. (2018) parten de la idea de que un juego móvil que permitiera a los jugadores aprender sobre las hierbas medicinales podría mejorar su conocimiento sobre las mismas. Por lo tanto, desarrollaron Herbopolis, un juego móvil sobre medicamentos a base de hierbas y se obtuvieron datos de usabilidad y efectividad sobre el juego a partir de un grupo piloto de jugadores. El objetivo del jugador en el juego era administrar la ciudad, expandir la línea de productos y maximizar los ingresos del comercio de hierbas. El guion gráfico se concibió con siete elementos principales del juego: mapa principal, granja, almacén, fábrica, empresa, tienda y sede internacional. El juego serio se encuentra orientado al aprendizaje y brinda servicios de concienciación con una interfaz agradable para motivar al usuario a través de la generación de consciencia, e incluye evaluaciones para validar la obtención de conocimientos a medida que se avanza en la ejecución del juego. Sin embargo, el prototipo no involucra retos que generen motivación a continuar con el juego ni tampoco cuenta recompensas que motiven a los usuarios a continuar aprendiendo mientras son recompensados por su rendimiento. Los trabajos anteriores permiten demostrar grandes fortalezas que ayudan a mostrar el enfoque que se tiene en cuanto al desarrollo de juegos serios dentro del campo de CSCL. Sin embargo, estas investigaciones también muestran algunas debilidades que deben afrontarse para mejorar los procesos de aprendizaje colaborativo utilizando juegos serios. Algunas de ellas, corresponden por ejemplo a que no consideran elementos importantes para la generación de la interacción entre los estudiantes, la detección de fallas cognitivas, la realización de evaluaciones adaptadas a las fallas de cada estudiante y la recomendación de recursos educativos (Park et al., 2012) con el objetivo de corregir las fallas identificadas. 
El objetivo de este artículo es entonces proponer y validar un modelo de juego serio colaborativo que integre agentes inteligentes de software, permitiendo la incorporación de funcionalidades de sensibilidad al contexto, es decir, utilizar características del estudiante y del entorno en el que este se desenvuelve para mejorar la identificación de fallas cognitivas. El enfoque de la investigación se centra entonces en la incorporación de herramientas lúdicas en entornos de aprendizaje colaborativo que permitan incentivar el interés de los estudiantes y mejorar la interacción de los integrantes del grupo. El juego colaborativo consiste en la elaboración de preguntas de evaluación a partir de contenidos previamente presentados en el aula de clase, estas preguntas son construidas en equipos y posteriormente almacenadas en una base de datos, utilizando el prototipo implementado. Posteriormente, se generan iteraciones de evaluación colaborativa que permiten contrastar los conocimientos obtenidos y detectar falencias al interior de los equipos, utilizando herramientas lúdicas. A partir de lo anteriormente mencionado, se pretende ofrecer una recomendación de recursos educativos proactiva que permita corregir dichas fallas mientras se genera consciencia del entorno en los participantes y se les motiva a participar de la actividad colaborativa. El modelo propuesto y el prototipo desarrollado permiten incorporar mecanismos de motivación, colaboración y participación en las actividades colaborativas, generando una interdependencia positiva que hace que necesario y fundamental la participación y el involucramiento de cada estudiante en la actividad, la cual es desarrollada por equipos.

\section{DESARROLLO DEL MODELO}

El modelo de juego serio desarrollado durante esta investigación, nace como una propuesta de software que integra tecnologías de agentes inteligentes y se propone como una herramienta lúdica para incrementar la comunicación, promover una competencia sana entre los participantes, lograr el involucramiento en el ambiente colaborativo y generar interdependencia positiva entre los integrantes de equipos de trabajo. De esta forma, se pretende la generación de entornos propicios para el potenciamiento del aprendizaje, teniendo como base los primeros cinco niveles de aprendizaje establecidos en la taxonomía de Bloom (Churches, 2008) los cuales son: retención, comprensión, aplicación, análisis y evaluación de conocimientos durante la ejecución del juego serio en entornos de aprendizaje colaborativo. Dicho modelo, surge a partir del esquema para el desarrollo de actividades colaborativas propuesto por Álvarez et. al (2018). En dicha investigación, se proponen las etapas E1: "Etapa lúdica para evaluación de conocimientos", A12: "Evaluación individual acumulativa" y B3: "Diagnóstico de fallas cognitivas" las cuales son un pilar fundamental para el diseño y desarrollo del modelo del juego serio propuesto. La Figura 1 presenta de forma específica y por roles, el diagrama del proceso correspondiente al modelo del juego serio colaborativo propuesto.

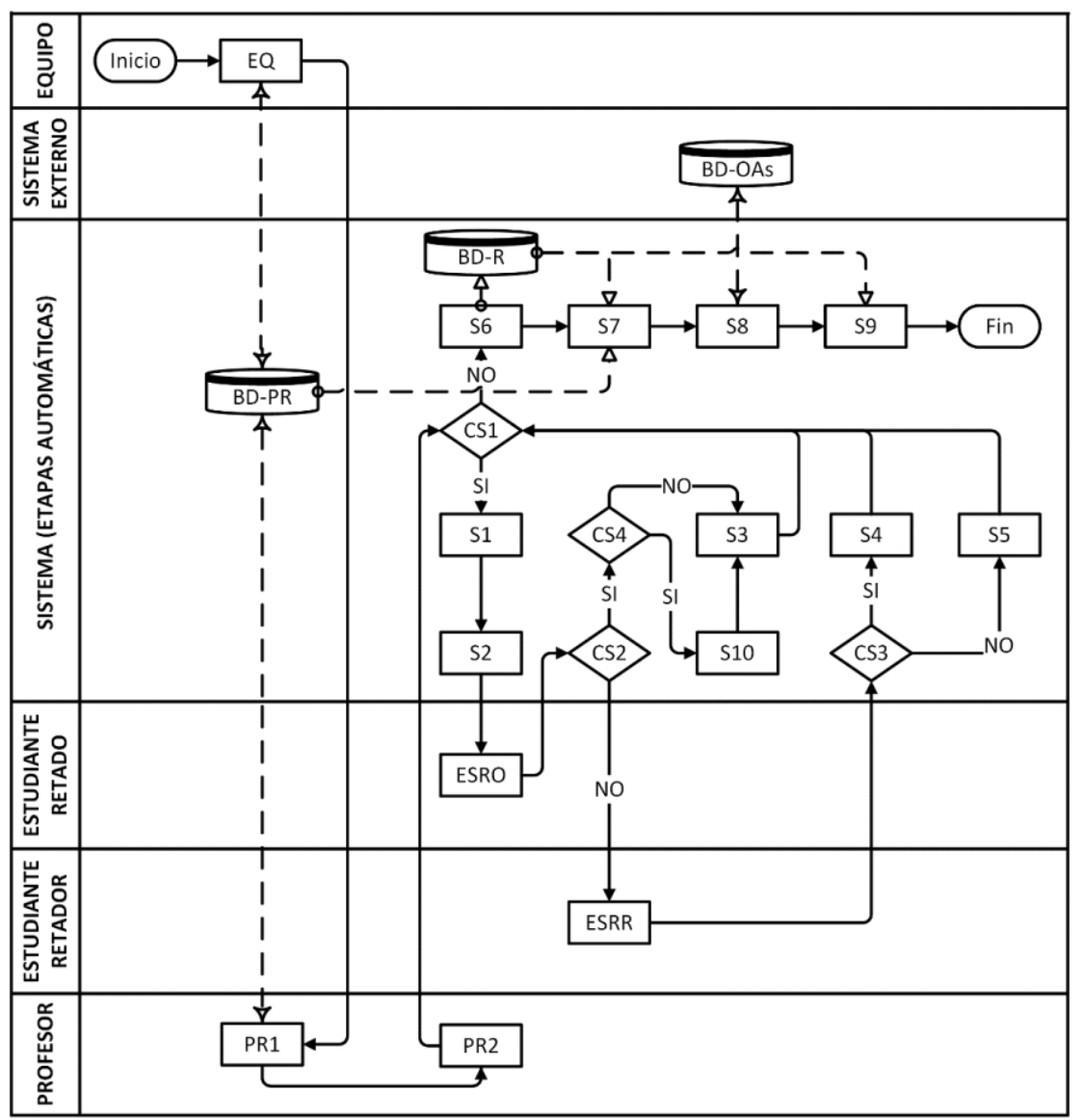

Fig. 1: Diagrama del proceso del juego serio colaborativo. 
El diagrama del proceso del juego serio colaborativo, consiste en una serie de etapas que tienen como objetivo el desarrollo de este enfoque de aprendizaje orientado al trabajo en equipo. En la Tabla 1 se detallan cada una de las etapas del juego serio y los agentes inteligentes que intervienen. Los agentes que componen el modelo colaborativo propuesto son: Agente Estudiante, Agente Profesor, Agente Gestor de Estadísticas, Agente Ontológico, Agente Evaluador y Agente Gestión Equipos.

Tabla 1: Descripción de las etapas del proceso correspondientes a la figura 1.

\begin{tabular}{|c|c|c|}
\hline Etapa & Agentes que intervienen & Descripción de la etapa \\
\hline BD-PR & $\begin{array}{l}\text { Ontológico, Profesor, } \\
\text { Evaluador, Estudiantes, } \\
\text { Gestión del equipo. }\end{array}$ & $\begin{array}{l}\text { Banco de preguntas. En esta base de datos se almacenan las preguntas construidas y con las } \\
\text { qcue se participará en el juego. }\end{array}$ \\
\hline BD-R & Evaluador, Ontológico. & $\begin{array}{l}\text { Base de datos de respuestas. En esta base de datos se almacenan cada una de las respuestas } \\
\text { seleccionadas durante la ejecución del juego. }\end{array}$ \\
\hline BD-OAS & Recomendador. & $\begin{array}{l}\text { Repositorio de objetos de aprendizaje. Es un repositorio externo donde se almacenan Objetos } \\
\text { de Aprendizaje los cuales son utilizados para realizar recomendaciones a partir de las fallas } \\
\text { diagnosticadas en la ejecución del juego. }\end{array}$ \\
\hline EQ & $\begin{array}{l}\text { Gestión del equipo, } \\
\text { Estudiantes. }\end{array}$ & $\begin{array}{l}\text { Etapa del proceso en la cual cada equipo realiza una construcción colaborativa de preguntas de } \\
\text { alto nivel. Dichas preguntas estarán acompañadas con respuesta de selección múltiple, } \\
\text { brindando así, algunas opciones incorrectas y una correcta. Para el inicio de esta etapa es } \\
\text { indispensable que los estudiantes se encuentren previamente organizados en equipos de } \\
\text { trabajo. }\end{array}$ \\
\hline PR1 & Profesor. & $\begin{array}{l}\text { El profesor realiza una categorización de preguntas por niveles de dificultad con el objetivo de } \\
\text { entregar insignias de acuerdo a unas reglas definidas al interior del juego. }\end{array}$ \\
\hline PR2 & Profesor. & El profesor da inicio a la etapa de preguntas. \\
\hline CS1 & Evaluador. & $\begin{array}{l}\text { El sistema verifica si hay preguntas que no se hayan jugado en ninguna de las rondas y ejecuta } \\
\text { el condicional siguiente: ¿Existen preguntas que no se hayan utilizado en alguna de las rondas? }\end{array}$ \\
\hline S1 & Evaluador, Estudiante. & $\begin{array}{l}\text { El sistema selecciona de forma aleatoria una pregunta, un estudiante del equipo donde se } \\
\text { construyó la pregunta (será un estudiante diferente a quien creó la pregunta y se conocerá de } \\
\text { aquí en adelante como estudiante retador) y un estudiante aleatorio de un equipo diferente } \\
\text { (designado como estudiante retado). }\end{array}$ \\
\hline S2 & Evaluador, Estudiante. & El sistema inicia una ronda de juego entre el estudiante retador y el estudiante retado. \\
\hline S3 & $\begin{array}{l}\text { Evaluador, Gestión del } \\
\text { equipo. }\end{array}$ & El sistema asigna un punto para el equipo al cual pertenece el estudiante retado. \\
\hline S4 & Evaluador. & El sistema no asigna ni resta puntos a ninguno de los dos equipos. \\
\hline S5 & $\begin{array}{l}\text { Evaluador, Gestión del } \\
\text { equipo. }\end{array}$ & El sistema resta un punto para el equipo al cual pertenece el estudiante retador. \\
\hline S6 & Evaluador. & $\begin{array}{l}\text { El sistema realiza la asignación de notas a cada equipo de acuerdo a la tabla de posiciones, } \\
\text { puntaje e insignias (reconocimientos) obtenidas. }\end{array}$ \\
\hline S7 & $\begin{array}{l}\text { Evaluador, Gestión del } \\
\text { equipo. }\end{array}$ & $\begin{array}{l}\text { El sistema genera de forma adaptada una evaluación individual acumulativa para cada } \\
\text { estudiante. Nota: La adaptación de la evaluación se realiza a partir de los siguientes criterios: } \\
\text { (1) Preguntas erradas por el estudiante; (2) Preguntas no contestadas por el equipo; (3) } \\
\text { Preguntas que no se jugaron, es decir, que no salieron en ninguna de las rondas del juego (si } \\
\text { aplica). }\end{array}$ \\
\hline S8 & Evaluador, Recomendador. & $\begin{array}{l}\text { El sistema califica la evaluación individual acumulativa de cada estudiante y dependiendo los } \\
\text { resultados, recomienda recursos educativos asociados a sus propias fallas detectadas. }\end{array}$ \\
\hline S9 & Gestor de Estadísticas. & El sistema realiza una presentación de estadísticas tanto al profesor como a los estudiantes. \\
\hline S10 & $\begin{array}{l}\text { Evaluador, Gestión del } \\
\text { equipo. }\end{array}$ & $\begin{array}{l}\text { El sistema asigna una insignia (reconocimiento) al equipo al cual pertenece el estudiante } \\
\text { retado. }\end{array}$ \\
\hline ESRO & Evaluador, Estudiante. & El estudiante retado debe responder correctamente la pregunta. \\
\hline ESRR & Evaluador, Estudiante. & El estudiante retador debe responder correctamente la pregunta. \\
\hline CS2 & Evaluador, Estudiante. & $\begin{array}{l}\text { El sistema verifica si el estudiante retado ha respondido la pregunta correctamente y ejecuta el } \\
\text { condicional siguiente: ¿Estudiante retado respondió la pregunta correctamente? }\end{array}$ \\
\hline CS3 & Evaluador, Estudiante. & $\begin{array}{l}\text { El sistema verifica si el estudiante retador ha respondido la pregunta correctamente y ejecuta el } \\
\text { condicional siguiente: ¿Estudiante retador respondió la pregunta correctamente? }\end{array}$ \\
\hline CS4 & Evaluador, Estudiante. & $\begin{array}{l}\text { El sistema verifica si el estudiante retado merece recibir una insignia (reconocimiento) y ejecuta } \\
\text { el condicional siguiente: ¿Estudiante retado cumple las condiciones para recibir una insignia? }\end{array}$ \\
\hline
\end{tabular}

\section{Agentes}

En la Tabla 2 se presenta funcionalidad y los roles que asumen los agentes durante la ejecución del juego serio colaborativo. Los principales agentes son los siguientes: Agente Estudiante, Agente Profesor, Agente Gestor de Estadísticas, Agente Ontológico, Agente Evaluador, Agente Gestión Equipos y Agente Recomendador. 
Tabla 2: Descripción de la funcionalidad y roles desempeñados por los agentes del juego colaborativo.

\begin{tabular}{|l|l|}
\hline Agente & Descripción de la funcionalidad y roles \\
\hline Agente Estudiante & $\begin{array}{l}\text { Se encarga de representar al estudiante en el ámbito de la plataforma, así como de administrar su perfil. Es un } \\
\text { agente de interfaz, que administra todas las interacciones (percepciones y/o acciones) entre el sistema y el } \\
\text { estudiante. }\end{array}$ \\
\hline Agente Profesor & $\begin{array}{l}\text { Representa al profesor dentro de la plataforma, lo asiste en el diseño instruccional de las actividades y la } \\
\text { calificación de informes. }\end{array}$ \\
\hline $\begin{array}{l}\text { Agente Gestor de } \\
\text { Estadísticas }\end{array}$ & $\begin{array}{l}\text { Presenta estadísticas de ejecución con el objetivo de generar consciencia a partir de la recopilación de información } \\
\text { del contexto. }\end{array}$ \\
\hline Agente Ontológico & $\begin{array}{l}\text { Administra la ontología de dominio específico propuesta por Álvarez et al. (2018), es decir, se encarga de poblar } \\
\text { las entidades y de realizar inferencias solicitadas por otros agentes. Dichas inferencias son generadas a partir del } \\
\text { uso de consultas y están relacionadas con la detección de fallas cognitivas, estructura de las actividades, recursos, } \\
\text { información puntual de los estudiantes y los equipos. }\end{array}$ \\
\hline Agente Evaluador & $\begin{array}{l}\text { Despliega las evaluaciones a los estudiantes, está encargado de la ejecución del juego, transfiere la información y } \\
\text { almacena los resultados en la BD (Base de Datos) del sistema. }\end{array}$ \\
\hline Agente Gestión Equipos & $\begin{array}{l}\text { Se encarga de la comunicación y transferencia de información con los agentes que representan a cada uno de los } \\
\text { estudiantes de los equipos. Además, este agente conoce los estudiantes que pertenecen a cada equipo. }\end{array}$ \\
\hline Agente Recomendador & $\begin{array}{l}\text { Tiene como objetivo entregar información al Agente Gestión Equipos para su posterior presentación. Por otro lado, } \\
\text { se encarga de recomendar recursos de aprendizaje a través del consumo de un servicio Web expuesto por un } \\
\text { sistema externo de administración de recursos de aprendizaje. Dicho sistema recibe las palabras clave inferidas } \\
\text { por el Agente Ontológico cuando se detectan fallas cognitivas. }\end{array}$ \\
\hline
\end{tabular}

Reglas y Definición de Conceptos Asociados al Juego Serio

Con el objetivo de brindar al usuario la información necesaria para comprender los elementos que componen el juego serio, en la Tabla 3 se detallan los conceptos principales manejados y las reglas asociadas al juego.

Tabla 3: Elementos principales del juego y reglas asociadas.

\begin{tabular}{|l|l|}
\hline Elemento del Juego & Reglas del Juego \\
\hline Número de integrantes de cada equipo & Debe estar entre 4 y 6 estudiantes \\
\hline Número mínimo de equipos & $\begin{array}{l}\text { Para poder llevar a cabo la actividad deben existir por lo menos tres (3) equipos } \\
\text { conformados. }\end{array}$ \\
\hline Escala de calificación & $\begin{array}{l}\text { La nota final asignada a cada equipo estará entre 0 y 5, donde 0 corresponde a la nota más } \\
\text { baja y 5 la más alta. }\end{array}$ \\
\hline Ronda & $\begin{array}{l}\text { Corresponde a cada una de las vueltas o series de turnos de los equipos que participan en } \\
\text { el juego. Una ronda se completa cuando todos los equipos juegan una vez como retados, } \\
\text { es decir, en cada ronda un equipo tiene que ser retado una sola vez. }\end{array}$ \\
\hline Número mínimo de rondas & $\begin{array}{l}\text { Corresponde al número de integrantes del equipo con mayor cantidad de estudiantes (ver } \\
\text { Ecuación 1) }\end{array}$ \\
\hline Número mínimo de preguntas por equipo & $\begin{array}{l}\text { Para poder llevar a cabo la actividad, cada equipo deberá construir como mínimo una } \\
\text { pregunta de más que el número de integrantes de su equipo (ver Ecuación 2) }\end{array}$ \\
\hline $\begin{array}{l}\text { Número mínimo de preguntas aprobadas por el } \\
\text { profesor en el banco para poder iniciar el juego }\end{array}$ & $\begin{array}{l}\text { Corresponde a la multiplicación entre el número mínimo de rondas y el número de equipos } \\
\text { (ver Ecuación3) }\end{array}$ \\
\hline Número máximo de rondas posibles & $\begin{array}{l}\text { Corresponde a la parte entera del resultado entre la división del número de preguntas en el } \\
\text { banco y el número de equipos (ver Ecuación 4) }\end{array}$ \\
\hline Cálculo de bonificaciones & $\begin{array}{l}\text { Se realiza para cada equipo a partir de la suma de la bonificación otorgada por las gemas } \\
\text { (ver Ecuación 5) }\end{array}$ \\
\hline Cálculo de nota final & $\begin{array}{l}\text { Se realiza para cada equipo a partir de los puntos de la tabla y la suma de la } \\
\text { bonificación otorgada por las gemas (ver Ecuación 6) }\end{array}$ \\
\hline $\begin{array}{l}\text { Cálculo de nota de la evaluación para cada } \\
\text { estudiante }\end{array}$ & $\begin{array}{l}\text { En vista de que es probable que las evaluaciones para cada estudiante queden de } \\
\text { diferente cantidad de preguntas, el cálculo de la nota de la evaluación deberá hacerse a } \\
\text { partir de una ecuación (ver Ecuación 7). }\end{array}$ \\
\hline
\end{tabular}

Las principales ecuaciones sobre las cuales se apoya el juego serio son las siguientes:

\#Rondasmin = \#IntegrantesEquipoMasGrande

\#PreguntasPorEquipomin = \#IntegrantesEquipos + 1

\#PreguntasBancomin=(\#Rondasmin X \#Equipos) + \#Equipos

\#Rondasmax $=($ \#PreguntasDisponibles - \#Equipos) / \#Equipos

Bonificación $=(\#$ Diamantes $X 0.2)+(\# R u b i e s$ X 0.1) $+($ \#smeraldas $\times 0.05)$

NotaFinalEquipo $=(5 \times$ PuntajeEquipo $) /$ \#RondasJugadas + Bonificación

NotaEvaluación $=(5 x$ \#PreguntasCorrectas $) /$ \#PreguntasContenidasEnEvaluación 
Adicionalmente, el juego serio debe cumplir las condiciones y modalidades que se describen en la Tabla 4.

Tabla 4: Condiciones y modalidades del juego.

\begin{tabular}{|l|l|}
\hline Condiciones y modalidades del juego & \multicolumn{1}{c|}{ Descripción } \\
\hline Condición 1 & Todos los estudiantes deben ser retados al menos una vez. \\
\hline Condición 2 & Todos los equipos son retados el mismo número de veces. \\
\hline Modalidad 1 (modo rápido) & Jugar la cantidad mínima de rondas posibles. \\
\hline Modalidad 2 (modo manual) & $\begin{array}{l}\text { Jugar una cantidad intermedia de rondas (entre el número máximo y el mínimo } \\
\text { de rondas posibles). }\end{array}$ \\
\hline Modalidad 3 (modo extendido): & Jugar la cantidad máxima de rondas posibles. \\
\hline
\end{tabular}

\section{Modelo de reconocimiento al buen desempeño}

En aras de incentivar la participación de los estudiantes en el juego, se propone un modelo de insignias (conocido en inglés como e-learning badges) compuesto por gemas tales como diamantes, rubíes y esmeraldas. Con este modelo, se pretende premiar ciertos comportamientos durante la ejecución del juego, evitando que a partir de las insignias se brinde ventajas únicamente a los mejores equipos (es decir, cualquier equipo estará en condiciones de ganar una insignia). En consecuencia, el modelo presentado evita que, por la asignación de las insignias, los equipos más deficientes siempre queden en las últimas posiciones de la tabla de resultados. Es necesario destacar que, para asignar las insignias, el profesor debe categorizar las preguntas en niveles de dificultad "bajo", "medio" y "alto", además de asignar los tiempos límite para responder cada pregunta. Dichas tareas deben realizarse antes del inicio de la ejecución del juego. De esta manera, la entrega de reconocimientos se realiza como se detalla en la Tabla 5.

Tabla 5: Insignias (reconocimientos) otorgadas por buen desempeño en el juego.

\begin{tabular}{|l|l|}
\hline Insignias & \multicolumn{1}{c|}{ Descripción } \\
\hline Diamante & $\begin{array}{l}\text { Se otorgará al equipo que tenga un acierto a la respuesta de una pregunta difícil en menos de } 15 \\
\text { segundos. }\end{array}$ \\
\hline Rubí & Se otorgará al equipo que tenga un acierto a la respuesta de una pregunta difícil \\
\hline Esmeralda: & $\begin{array}{l}\text { Se otorgará al equipo que tenga un acierto a la respuesta de una pregunta de nivel medio en menos de } \\
15 \text { segundos. }\end{array}$ \\
\hline
\end{tabular}

Al finalizar la ronda de preguntas del juego, se presentará la tabla de posiciones, se calculará la nota para cada equipo y se asignará una bonificación a partir del intercambio de las gemas obtenidas por un aumento en la nota que recibirá cada equipo según su posición la tabla (dicho aumento no podrá sobrepasar en ningún caso el tope de la escala de calificación). La descripción de las bonificaciones que se pueden conseguir dependiendo de la insignia recibida se presenta en la Tabla 6.

Tabla 6: Bonificaciones según Insignias recibidas.

\begin{tabular}{|l|l|}
\hline Bonificaciones & \multicolumn{1}{c|}{ Descripción } \\
\hline Diamante & Cada gema de este tipo equivale a un aumento de 0.2 en la nota final. \\
\hline Rubí & Cada gema de este tipo equivale a un aumento de 0.1 en la nota final. \\
\hline Esmeralda: & Cada gema de este tipo equivale a un aumento de 0.05 en la nota final. \\
\hline
\end{tabular}

Con el fin de validar el modelo propuesto, se construyó un prototipo de juego serio haciendo uso de diferentes tecnologías, así: para el montaje de la base de datos se utilizó el motor MySQL, para el desarrollo del juego en el Frontend se hizo uso del Framework Laravel versión 5.6 con vistas implementadas en Blade. Dicho Frontend se comunica por medio de servicios Web REST expuestos a través de una API desarrollada en JAVA con JADE para el manejo de los agentes. El juego serio colaborativo fue denominado COLEGA. EI nombre brindado proviene de las siglas en inglés para Collaborative Learning Game, además, hace apología a su significado el cual se encuentra asociado con la amistad o con las personas que ejercen una misma actividad, en este caso, una actividad enmarcada en un curso académico.

En la Figura 2, se presenta una de las interfaces del prototipo del juego serio colaborativo, esta corresponde a la interfaz con más interacción, dinamismo, y complejidad de COLEGA. Es importante destacar que dicha pantalla está compuesta por tres componentes. El primero se encarga de presentar la información relacionada con el tiempo restante para responder a la pregunta en ejecución, la tabla de posiciones actualizada en tiempo real y la tabla de insignias por cada uno de los equipos. El segundo componente de la interfaz, presenta el turno y la ronda en ejecución actual, la pregunta en juego con sus opciones de respuesta y los botones para responder $u$ omitir la pregunta. Finalmente, el tercer componente se encarga de presentar las notificaciones y alarmas utilizando cuadros de texto emergentes que permiten motivar e informar al estudiante constantemente. Para esta pantalla, es importante destacar que existe una división de los componentes 
presentados en pantalla, así: en el lado izquierdo se presenta el contador de tiempo, y las tablas de posiciones e insignias por equipo. Adicionalmente, en el lado derecho y central de la interfaz se presenta la información del turno y ronda en ejecución, la pregunta en juego con sus opciones de respuesta, las notificaciones y alarmas, y los botones para responder $u$ omitir la pregunta. Los elementos que componen esta pantalla se actualizan constantemente con el cambio de turno.

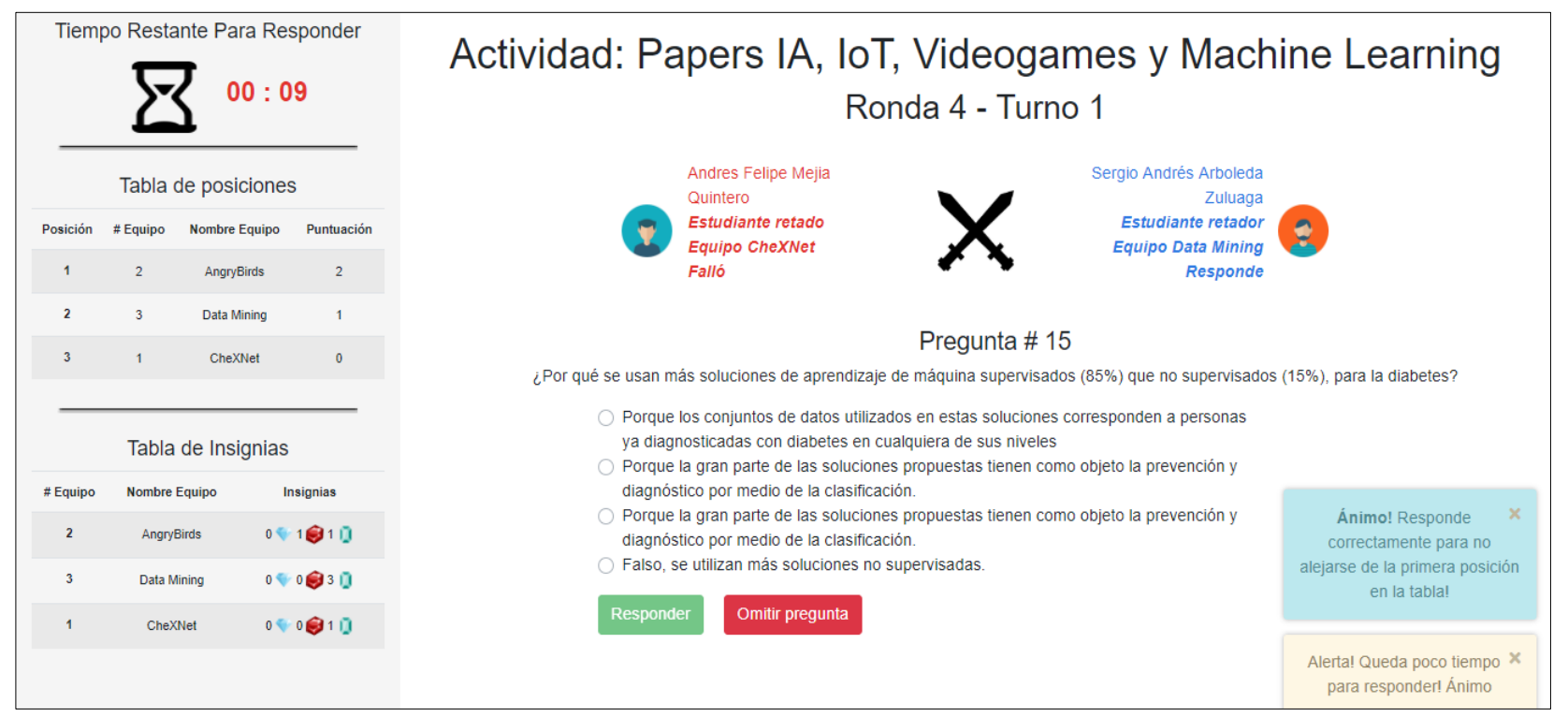

Fig. 2: Interfaz principal de COLEGA.

\section{RESULTADOS}

Para realizar el proceso de validación, se construyó y desplegó un estudio de caso en un ambiente controlado, específicamente al interior del curso de Inteligencia Artificial dictado en la Facultad de Minas de la Universidad Nacional de Colombia - Sede Medellín. El estudio de caso contó con un profesor, 15 estudiantes divididos en 3 equipos de trabajo los cuales interactuaron con COLEGA y recorrieron el flujo del juego serio colaborativo hasta finalizarlo. Adicionalmente, los estudiantes construyeron un total de 18 preguntas de alto nivel y fueron asignados objetivos educacionales a las mismas por parte del profesor. Finalmente se simuló un repositorio de OA al interior del sistema y fue poblado con 25 OA reales con temáticas asociadas a las preguntas creadas.

Posteriormente, se consideraron cinco enfoques diferentes a partir de una encuesta de percepción realizada a los estudiantes que participaron en el estudio de caso, así: 1) se validó de forma general el modelo y el prototipo implementados; 2) se validó la efectividad en la detección de fallas y asignación de recursos educativos recomendados para cada estudiante; 3) se validó la funcionalidad y el nivel de adaptación de la evaluación final acumulativa generada para cada estudiante; 4) se realizó una validación de los servicios de concienciación implementados; y 5) se validó la percepción por parte de los estudiantes acerca de los conocimientos obtenidos haciendo uso del juego en comparación con el uso de una metodología tradicional de aprendizaje. Además, para complementar la validación del prototipo, fueron realizadas encuestas de percepción y satisfacción a los estudiantes involucrados en el estudio de caso desarrollado. Dichas encuestas se realizaron a través de la plataforma Google Forms una vez los estudiantes finalizaron la interacción con el juego serio, esto con el objetivo de indagar sobre cada uno de los elementos presentados y percibidos por el usuario. Las preguntas y resultados de la encuesta son presentados a continuación. Es importante aclarar que no se realizó una evaluación de conocimientos a otros equipos que no participaron en la ejecución del juego colaborativo.

\section{Evaluación general del modelo y prototipos implementados}

Tal como se mencionó previamente, se utilizaron encuestas de percepción y satisfacción con el fin de validar el modelo propuesto. En dichas encuestas, se realizaron tres preguntas relacionadas específicamente con el modelo propuesto y el prototipo implementado, logrando obtener los resultados presentados en la Figura 3. Los resultados de percepción muestran un alto porcentaje de aceptación del modelo propuesto y del prototipo implementado, ya que, la totalidad de estudiantes (100\%) considera que el prototipo es fácil de utilizar para apoyar actividades en entornos de aprendizaje colaborativo, y que además se sienten motivados a interactuar y compartir conocimientos. Por otra parte, el $73 \%$ de estudiantes considera que el prototipo aporta considerablemente al trabajo colaborativo, mientras que ninguno de los estudiantes manifestó ausencia de elementos de interacción dentro del prototipo. 
$¿$ Considera que el prototipo es fácil de utilizar como una herramienta para apoyar el aprendizaje colaborativo?

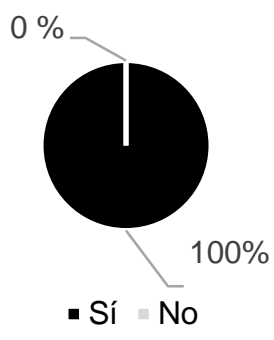

¿El prototipo genera motivación a interactuar, a compartir conocimientos y conocer todas las respuestas al interior de su equipo?

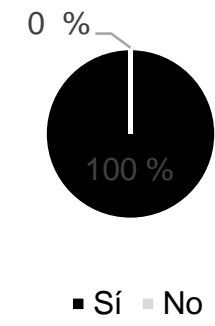

¿ El prototipo genera motivación a interactuar, a compartir?

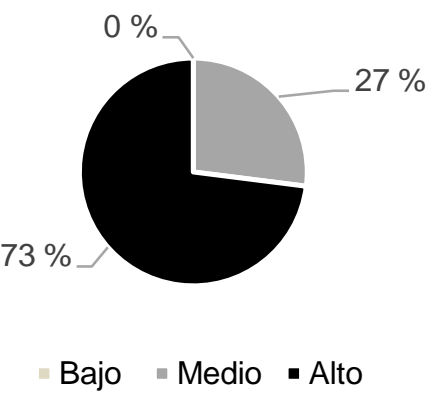

Fig. 3: Medición de resultados de la encuesta de percepción asociada con la validación general del modelo y prototipo implementados.

Los resultados acerca del desempeño presentados en la última pregunta fueron contrastados con los estudiantes encuestados y se evidenció que el desempeño medio del prototipo correspondió a que, durante la ejecución del estudio de caso todos los estudiantes debían desplazarse a responder en el mismo computador y no podían hacerlo cada uno desde su propio equipo. Lo anterior, fue debido a que, en esta etapa de validación, el desarrollo no contempló la gestión de la comunicación y sincronización entre dispositivos para lanzar la pantalla de respuesta únicamente a quien le correspondía responder en un turno. Esto, debido a que implicaba un desarrollo mucho más complejo y no era el objetivo del desarrollo de esta investigación.

\section{Efectividad en la detección de fallas y asignación de recursos educativos recomendados para cada estudiante}

De igual manera a como se realizó la validación del modelo y del prototipo, se utilizaron las encuestas de percepción y satisfacción para evaluar la efectividad en la detección de fallas y asignación de recursos educativos recomendados para cada estudiante. Para este caso, se realizaron 2 preguntas específicamente relacionadas con el diagnóstico de fallas y los recursos recomendados, logrando obtener los resultados presentados en la Figura 4. En este caso, los resultados de percepción muestran un comportamiento que es necesario explicar en detalle, sin embargo, existió un alto porcentaje de éxito del modelo y del prototipo en cuanto a detección y recomendación de fallas se refiere. Revisando la primera pregunta, se contrastaron los resultados obtenidos con la opinión personal de cada uno de los estudiantes encuestados y se evidenció que el $27 \%$ de ellos no recibió detección de fallas cognitivas por parte de COLEGA, sin embargo, este comportamiento fue debido a que estos estudiantes no cometieron errores en ninguna de las preguntas que el sistema les asignó a ellos. En el mismo sentido, esta situación impactó directamente la recomendación de recursos a estos estudiantes, lo que explica que el mismo porcentaje no haya recibido ninguna recomendación por parte de COLEGA.

¿Considera que las fallas detectadas por el sistema realmente corresponden a sus deficiencias cognitivas relacionadas con los conceptos asociados a la actividad ejecutada?

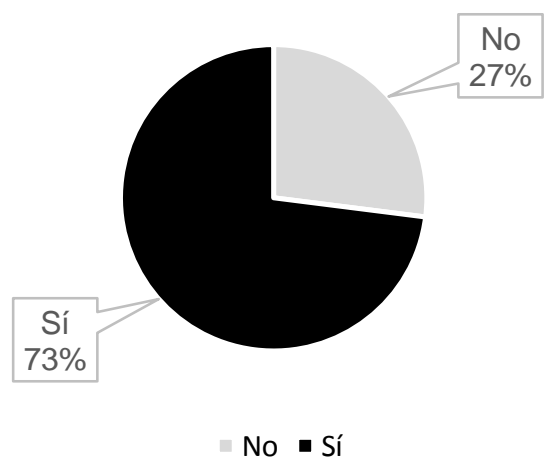

¿Considera que los recursos recomendados se encuentran asociados con sus debilidades o fallas cognitivas?

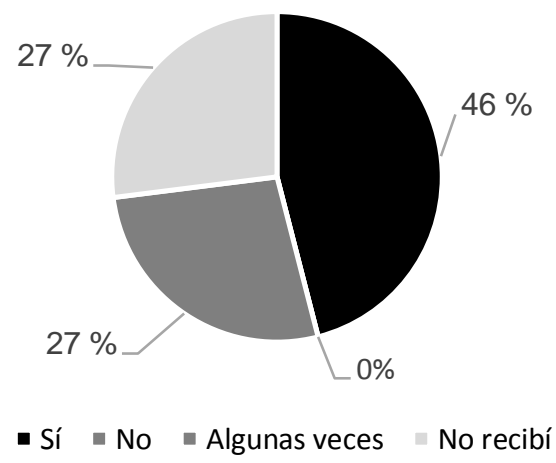

Fig. 4: Medición de resultados de la encuesta de percepción asociada con la efectividad en la detección de fallas y asignación de recursos educativos recomendados para cada estudiante. 


\section{Nivel de adaptación de la evaluación final acumulativa generada para cada estudiante}

Para realizar la validación de esta sección, se incluyeron 2 preguntas específicamente relacionadas con el nivel de adaptación de la evaluación final acumulativa generada por COLEGA de forma automática para cada uno de los estudiantes involucrados en la ejecución del sistema. Se lograron obtener los resultados presentados en la Figura 5.

\section{Considera que la generación de la evaluación final se encuentra...}

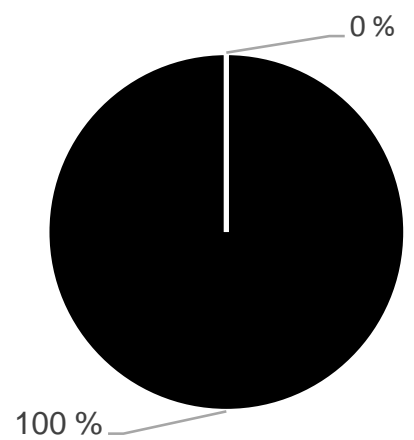

Adaptada a sus fortalezas

- Adaptada a las debilidades de su equipo

No se encuentra adaptada
En caso que considere que la evaluación SI se encuentra adaptada, ¿Qué tan adaptada considera que es la evaluación?

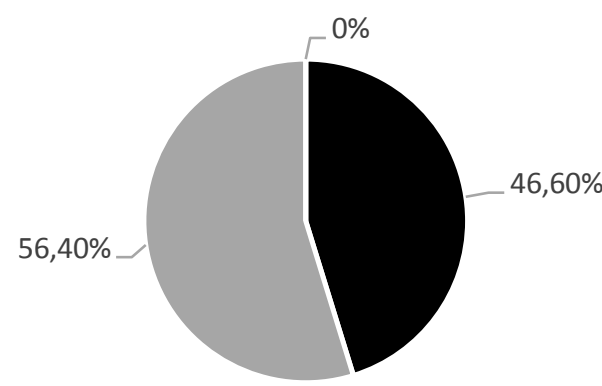

- Alto nivel de adaptación

- Medio nivel de adaptación

Bajo nivel de adaptación

Fig. 5: Medición de resultados de la encuesta de percepción asociada con la efectividad en la detección de fallas y asignación de recursos educativos recomendados para cada estudiante.

A partir de los resultados obtenidos con las preguntas anteriores, se puede identificar que la totalidad de los estudiantes que participaron en el estudio de caso, realmente notaron y percibieron que la evaluación acumulativa se encontraba adaptada a cada uno de ellos y que ésta, estaba construida a partir de preguntas asociadas a sus debilidades o que no les había tocado responder en su equipo en ninguno de los turnos de juego. Por otro lado, tal como se observa en las respuestas de la segunda pregunta, el $53 \%$ de los estudiantes indicaron un nivel medio de adaptación, mientras que el $47 \%$ manifestaron percibir un nivel alto de adaptación.

Evidentemente, aunque ningún estudiante manifestó percibir bajo nivel de adaptación de la evaluación, dicha distribución de porcentaje es un comportamiento explicado por la poca cantidad de preguntas construidas por los equipos antes de iniciar el juego; es decir, si bien es cierto que se cumplió con la cantidad mínima de preguntas que indica el juego en sus reglas, es recomendable que exista un mayor número de preguntas en el banco para que COLEGA cuente con más opciones para adaptar la evaluación a cada estudiante.

\section{Evaluación de los servicios de concienciación}

Esta sección presenta la validación de los servicios de concienciación implementados, para lograrlo se incluyeron 8 preguntas específicamente relacionadas con los servicios de concienciación integrados en COLEGA. Se lograron obtener los resultados presentados en la Figura 6. Los resultados obtenidos en las preguntas anteriores evidencian el alto porcentaje de aceptación y utilidad de los servicios de concienciación ofrecidos por COLEGA, lo anterior en vista de que de manera específica el $80 \%$ de los estudiantes manifestaron utilidad y entendieron la utilidad de los servicios. Adicionalmente, se evidenció que hubo dificultad por parte de los estudiantes en el hecho de entender el funcionamiento de los servicios, sin embargo, al contrastar los resultados de la pregunta asociada a este ítem con la opinión en conjunto de los estudiantes, quedó claro que la falta de entendimiento estaba asociada al desconocimiento del concepto de concienciación.

A partir del análisis de los resultados anteriores, puede evidenciarse que, aunque es clara la utilidad de los servicios de concienciación dentro de COLEGA, los servicios relacionados con la tabla de posiciones, tabla de insignias, y alarmas y notificaciones, pueden presentar problemas en la claridad de la información. Además, a partir de lo percibido durante la ejecución del estudio de caso, se pudo apreciar que los estudiantes al estar inmersos en su turno y buscar la respuesta de la pregunta no son muy conscientes de lo que sucede en la interfaz. Finalmente, es importante destacar que los servicios mejor calificados fueron: estadísticas de ejecución (100\%) y recursos recomendados (93\%). 


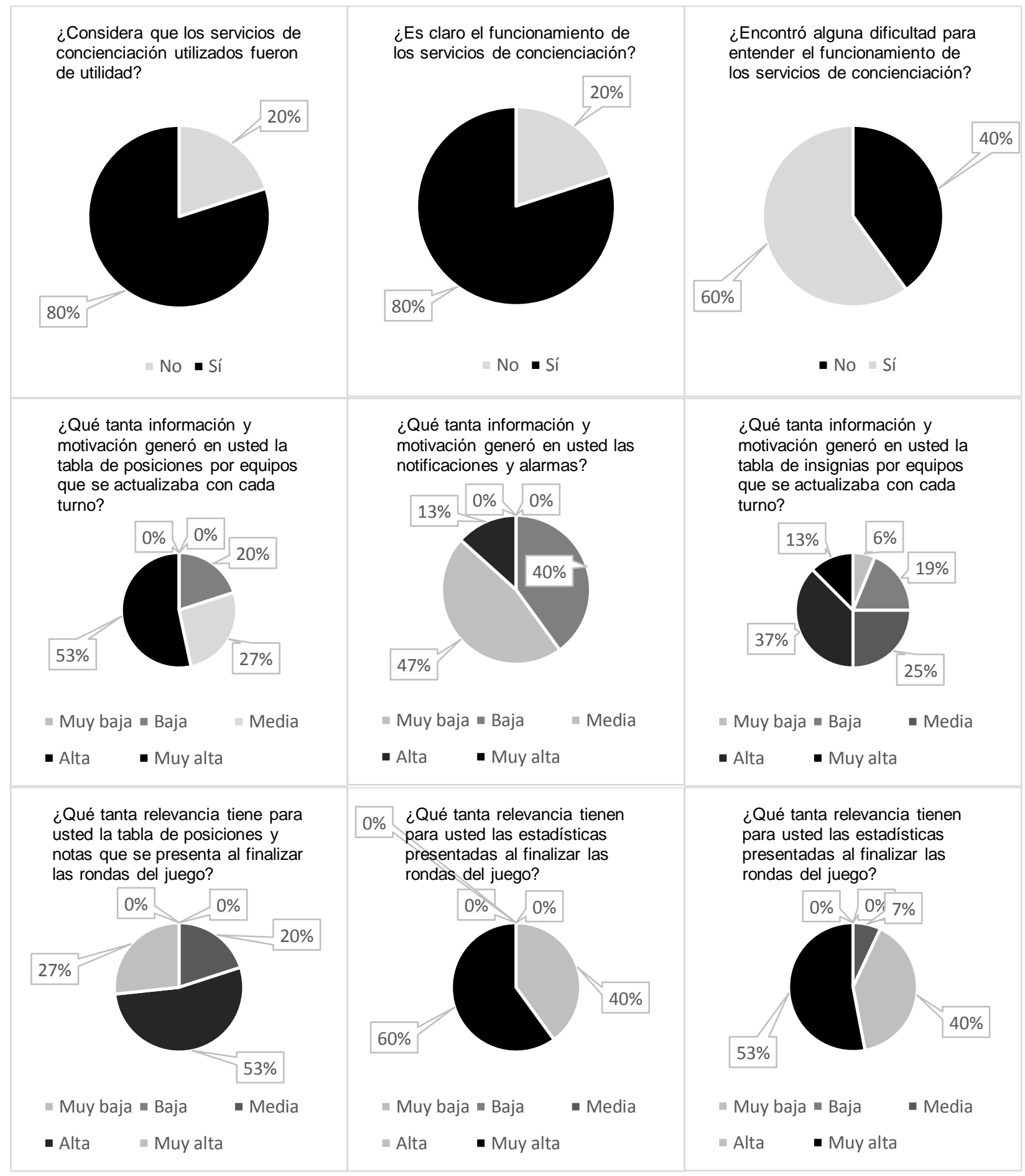

Fig. 6: Medición de resultados de la encuesta de percepción asociada con la validación de los servicios de concienciación.

\section{Evaluación de conocimientos obtenidos haciendo uso del juego}

Aunque no se realizó una evaluación de conocimientos a otros equipos que no participaron en la actividad para comparar los conocimientos obtenidos con los dos modelos, sí se incluyó una pregunta relacionada con el nivel de adquisición de conocimientos en la encuesta de percepción para conocer la opinión de los estudiantes. Se obtuvieron los resultados presentados en la Figura 7. A partir de los resultados obtenidos con la pregunta asociada a la Figura 7, se puede identificar que la totalidad de los estudiantes que participaron en el estudio de caso están de acuerdo con que de esta manera aprenden mucho más. Además, los estudiantes manifestaron que, al sentirse más motivados, su nivel de retención de información se ve aumentado y que los modelos tradicionales de aprendizaje no generan tanta motivación. A continuación, se presenta el resumen de resultados de la validación mencionada. 
¿Considera que el modelo propuesto puede aumentar el nivel de conocimientos adquiridos en comparación con las metodologías tradicionales de aprendizaje?

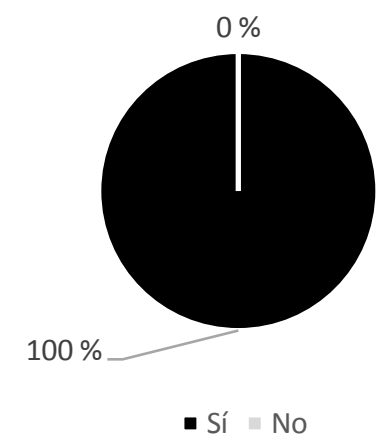

Fig. 7: Medición de resultados de la encuesta de percepción asociada con el nivel de adquisición de conocimientos de los estudiantes en comparación con las metodologías tradicionales de aprendizaje.

\section{DISCUSIÓN}

Al comparar el enfoque presentado en este artículo con el de otros investigadores se puede afirmar que este trabajo de investigación incluye la incorporación de varias técnicas computacionales a saber: un juego serio para aprendizaje colaborativo que integra agentes inteligentes de software, permitiendo la incorporación de funcionalidades de sensibilidad al contexto, es decir, utilizar características del estudiante y del entorno en el que este se desenvuelve para mejorar la identificación de fallas cognitivas. A partir de lo anteriormente mencionado, se buscó ofrecer una recomendación de recursos educativos proactiva que permita corregir dichas fallas mientras se genera consciencia del entorno en los participantes y se les motiva a participar de la actividad colaborativa.

Aunque Bennane (2013) propone un sistema multi-agente SMAASA el cual integra lógica difusa para la automatización del apoyo de los alumnos en una plataforma de aprendizaje colaborativo en línea (Collaborative e-learning Platform) sin embargo, no se incluyen los aspectos lúdicos de los juegos serios con el fin de generar mayor interacción, poder compartir y generar nuevos conocimientos, lo cual permite que los estudiantes se motiven por medio de las herramientas ofrecidas por la plataforma para colaborar y adquirir nuevos conocimientos. Adicionalmente, en este trabajo no se recomiendan recursos educativos que permitan corregir fallas cognitivas.

Por su parte, Samadi et al. (2018) aunque proponen un sistema de preguntas y respuestas QAS (por sus siglas en inglés para Question Answering System) el cual posee un enfoque con agentes sintácticos y semánticos para responder preguntas en plataformas de aprendizaje en línea (e-learning), sin embargo, los autores no manejan los aspectos colaborativos de trabajo en equipo para la generación de preguntas, ni utilizan juegos serios con el fin de motivar el aprendizaje. Tampoco se propone en esta investigación la utilización de mecanismos de recomendación de recursos educativos para solventar fallas de aprendizaje.

Finalmente, aunque González-González et al. (2019) incluyen aspectos lúdicos dentro de su propuesta para desarrollar un sistema inteligente de rehabilitación basado en el videojuego de ejercicio TANGO:H (por sus siglas en inglés para Tangible Goals: Health) la cual puede ser usada para apoyar el proceso de rehabilitación de niños hospitalizados, sin embargo, no considera aspectos colaborativos donde varios niños hospitalizados pudieran interactuar y así generar motivación entre ellos dentro de un ambiente lúdico colaborativo. Adicionalmente, esta investigación no considera la detección de fallas en el proceso de rehabilitación de los niños hospitalizados, pero en cambio, sí utiliza el historial del usuario, para seleccionar nuevos ejercicios gamificados lo cual se puede asociar a la realimentación de conocimientos (i.e. actividades de rehabilitación).

\section{CONCLUSIONES}

A partir de los resultados obtenidos con la implementación del juego serio colaborativo COLEGA, y luego de realizar la validación del prototipo se logra concluir que:

i) El modelo de juego serio basado en agentes inteligentes y aprendizaje colaborativo brinda mecanismos que motivan la colaboración y participación en la actividad, mientras que se genera una interdependencia positiva que hace que sea necesario y fundamental la participación y el involucramiento de cada estudiante en la actividad, la cual es desarrollada por equipos. 
ii) La integración de las tecnologías propuestas aporta de manera positiva a la generación, transferencia y retención del conocimiento por parte de los estudiantes. Además, la integración de los elementos tecnológicos tales como servicios sensibles al contexto contribuye a generar un ambiente colaborativo más propicio y ameno para el aprendizaje.

iii) Los resultados obtenidos muestran que la integración de las tecnologías propuestas aporta de manera positiva a la generación, transferencia y retención del conocimiento por parte de los estudiantes. Además, el modelo al integrar enfoques lúdicos y colaborativos brinda mecanismos que motivan la colaboración y participación en las actividades con miras a mejorar el aprendizaje.

\section{AGRADECIMIENTOS}

La investigación presentada en este artículo fue financiada parcialmente por la beca de Doctorado de COLCIENCIAS, otorgada a Oscar Salazar a través de la Convocatoria 761 "Convocatoria Nacional de Jóvenes Investigadores e Innovadores 2016".

\section{REFERENCIAS}

Alzaza, N.S., y Yaakub, A. R., Students' Awareness and Requirements of Mobile Learning Services in the Higher Education Environment, American Journal of Economics and Business Administration, 3 (1) 95-100 (2011).

Aguilera, A., Fúquene, C.A., y Ríos, W.F., Aprende jugando: el uso de técnicas de gamificación en entornos de aprendizaje, Revista IM-Pertinente, 2(1) 125-143 (2014).

Acevedo, C.P., Arciniegas, J.L., García, X. y Perrinet, J., Proceso de Adaptación de una Aplicación de e-aprendizaje a taprendizaje, Información Tecnológica, 21(6) 27-36 (2010).

Álvarez, S., Salazar, O.M., y Ovalle, D.A., Modelo basado en Agentes para la Detección de Fallas Cognitivas en Entornos de Aprendizaje Colaborativo, Información Tecnológica, 29(5) 289-298 (2018).

Bennane, A., Adaptive Educational Software by Applying Reinforcement Learning, Informatics in Education, 12(1) 13-27 (2013).

Briede, J.C., Leal, I.M., Mora, M.L., y Pleguezuelos, C.S., Propuesta de Modelo para el Proceso de EnseñanzaAprendizaje Colaborativo de la Observación en Diseño, utilizando la Pizarra Digital Interactiva (PDI), Formación Universitaria, 8(3), 15-26 (2015).

Caicedo, C., y Weiss, M., The viability of spectrum trading markets, IEEE Communications Magazine, 49(3) 46-52 (2011).

Carrera, Á., Iglesias, C.A., García-Algarra, J., y Kolařík, D., A real-life application of multi-agent systems for fault diagnosis in the provision of an Internet business service, Journal of Network and Computer Applications, vol. 37, 146-154 (2014).

Ee, R.W.X., Yap, K.Z., y Yap, K.L.Y, Herbopolis - A mobile serious game to educate players on herbal medicines, Complementary Therapies in Medicine, 39, 68-79 (2018).

Elimelech, O., Stern, R., Kalech, M., y Bar-Zeev, Y., Diagnosing resource usage failures in multi-agent systems, Expert Systems with Applications, vol 77, 44-56 (2017).

González-González, C.S., Toledo-Delgado, P.A., Muñoz-Cruz, V., y Torres-Carrion, P.V., Serious games for rehabilitation: Gestural interaction in personalized gamified exercises through a recommender system, Journal of Biomedical Informatics, 97, 103266 (2019).

Hainey, T., Westera, W., Connolly, T.M., Boyle, L., Baxter, G., y Beeby, R.B., Students' attitudes toward playing games and using games in education: comparing Scotland and the Netherlands, Computers \& Education, vol 69, 474-484 (2013).

Hernández-Horta, I.A., Monroy-Reza, A., y Jiménez-García, M., Aprendizaje mediante Juegos basados en Principios de Gamificación en Instituciones de Educación Superior, Formación Universitaria, 11(5) 31-40 (2018).

Hernández, M.R., Rodríguez, V.M., Parra, F.J., y Velázquez, P., Las Tecnologías de la Información y la Comunicación (TICs) en la Enseñanza-Aprendizaje de la Química Orgánica a través de Imágenes, Juegos y Videos, Formación Universitaria, 7(1), 31-40 (2014).

Lhafiane, F., Elbyed, A., y Bouchoum, M., Multi Agent System Architecture Oriented Prometheus Methodology Design for Reverse Logistics, International Journal of Computer, Electrical, Automation, Control and Information Engineering, 9(8) 1914-1920 (2015).

Mizukoshi, K., Oshima, T., Mizuochi, Y., y Yatsushiro, K., A portable CSCL system "edutab box" with an autonomous wireless network. http://doi.org/10.1109/SII.2016.7844071, IEEE- Explore, SII 2016 - IEEE/SICE International Symposium on System Integration, 640-645 (2016).

Ovalle, D.A., Salazar, O.M., y Duque, N.D., Modelo de recomendación personalizada en cursos virtuales basado en computación ubicua y agentes inteligentes, Información Tecnológica, 25(6) 131-142 (2014).

Park, D.H., Kim, H.K., Choi, I.Y., y Kim, J.K., A literature review and classification of recommender systems research, Expert Systems with Applications, 39(11) 10059-10072 (2012). 
Proffitt, R., Sevick, M., Chang, C-Y., y Lange, B., User-centered design of a controller-free game for hand rehabilitation, Games for health journal, 4(4) 259-264 (2015).

Rego, P.A., Moreira, P.M., y Reis, L.P., A serious games framework for health rehabilitation, International Journal of Healthcare Information Systems and Informatics, 9(3) 1-21 (2014).

Samadi, A., Hanaa, E.F., Qbadou, M., Youssfi, M., y Akef, F., A syntactic and semantic multi-agent based question answering system for collaborative e-learning. http://doi.org/10.1109/ICOA.2018.8370588, IEEE- Explore, ICOA 2018, 14 (2018).

Terzidou, T., Tsiatsos, T., Miliou, C., y Sourvinou, A., Agent Supported Serious Game Environment, IEEE Transactions on Learning Technologies, 9(3) 217-230 (2016). 
